\title{
The production of very-long-chain PUFA biosynthesis in transgenic plants: towards a sustainable source of fish oils
}

\author{
Johnathan A. Napier* and Olga Sayanova \\ Crop Performance and Improvement Division, Rothamsted Research, Harpenden, Herts AL5 2JQ, UK
}

\begin{abstract}
There is now considerable evidence of the importance of $n$-3 long-chain PUFA in human health and development. At the same time, the marine fish stocks that serve as the primary sources of these fatty acids are threatened by continued over-exploitation. Thus, there is an urgent need to provide a sustainable alternative source of the $n$ - 3 long-chain PUFA normally found in fish oils. The possibility of using transgenic plants genetically engineered to synthesise these important fatty acids has recently been demonstrated. The approaches taken to realise this outcome will be discussed, as will their prospects for providing a sustainable resource for the future.
\end{abstract}

Desaturase: Elongase: PUFA: Transgenic plants: Sustainability

There is now good evidence from many scientific publications that the human diet not only plays a vital role in normal development, but is also central to robust health; conversely, poor diet (and hence nutrition) can lead to the acquisition of, or progression to, diseased states (for recent reviews, see Go et al. 2003; Paoloni-Giacobino et al. 2003). Controlled clinical studies have provided a means by which important components of the human diet can be identified and their efficacy tested (von Schacky, 2003; GISSI-Prevenzione Investigators, 1999). In addition, the importance of genetic make-up in the responsiveness of an individual or population to any particular dietary regimen ('diet-gene' interactions) has also emerged recently (German et al. 2003), and has been underpinned by the completion of the human genome sequence and the parallel genotyping of large numbers of individuals. Such studies point towards considerable genetic variation amongst mankind globally. It has been estimated that there are in excess of three million polymorphisms in the human population, with these variations mainly taking the form of single nucleotide polymorphisms (Zhao et al. 2003). Thus, it is also important to consider these genetic components in attempts to understand and modulate human health through optimised diet and nutrition (Shastry, 2002).

An excellent example of the impact of nutrition that is of particular relevance to the topic of the present review is demonstrated by the work of Bang, Dyerberg and colleagues (Bang et al. 1976; Dyerberg \& Bang, 1982).
These Swedish researchers have carried out pioneering studies on the prevalence of CVD in Inuit (Eskimo) communities whose diet is rich in oily fish (Bang et al. 1976). The resulting data led the authors to suggest the need for large-scale dietary intervention studies to determine the efficacy of high-fish-oil diets rich in $n$-3 longchain (LC) PUFA (Dyerberg \& Bang, 1982). It is as a result of such subsequent studies that it is now generally considered that $n-3$ LC PUFA are not only a pivotal component of the human diet, but they are also actively beneficial to health, providing a protective role against a range of diseases (and progressions to diseased states; for reviews, see Simopoulos, 2000; Nugent, 2004). Such human pathologies include not only CVD but also metabolic syndrome, which is a collective description for a number of indicators of risk of type 2 diabetes and obesity (Nugent, 2004). These indicators (such as high blood pressure, high plasma triacylglycerols and abnormal fasting blood glucose) are increasingly more prevalent in Western populations, and while the precise (presumptively multi-factorial) causes of these symptoms are unclear, they are of considerable concern. In particular, the apparent increases in both obesity and type 2 diabetes represent a major public health problem (Graham et al. 2004; Nugent, 2004). However, the observation that $n-3$ LC PUFA can provide some extent of pan-genotypic protection from these diseases has focused research on the biosynthesis and production of these important fatty acids (Graham et al. 
2004). It is also noteworthy that there is now good genetic evidence (i.e. polymorphisms in biosynthetic genes) to link $n$-3 LC PUFA with a number of human diseases such as retinal dystrophy (Klaver \& Allikmets, 2003).

In particular, two $n-3$ LC PUFA found in fish oils, EPA $(20: 5 n-3)$ and DHA (22:6n-3), are known to be important in helping to prevent both CVD and metabolic syndrome (Graham et al. 2004; Nugent 2004). It has been shown that a modest intake of fatty fish may reduce mortality in men who have recovered from myocardial infarction (Burr et al. 1989). The GISSI-Prevenzione trial (GISSIPrevenzione Investigators, 1999) has confirmed these findings in a large intervention study of patients surviving myocardial infarction; relatively modest $(<1 \mathrm{~g} / \mathrm{d})$ consumption of $n-3$ LC PUFA markedly lowers the risk of CVD and death. Dietary EPA and DHA reduce blood coagulation, blood lipids and blood pressure and have antiarrhythmic effects in adults. They may increase HDLcholesterol, suppress inflammatory and allergic processes and benefit mental disorders (Hu et al. 2001; von Schacky 2003).

\section{Long-chain PUFA in the human diet}

The synthesis of LC PUFA is usually carried out by an aerobic process involving the sequential introduction of double bonds and $\mathrm{C}_{2}$ elongation of a fatty acid substrate (an anaerobic process similar to the processive synthesis of polyketides has been described in several marine microorganisms; Metz et al. 2001; Napier, 2002). In mammals LC PUFA biosynthesis is dependent on the dietary intake of linoleic acid $(18: 2 n-6 ;$ LA) and $\alpha$-linolenic acid (18:3n-3; ALA; hence their designation as essential fatty acids), since higher animals have lost the capacity to synthesise these two fatty acids (Wallis et al. 2002). Crucially, the efficiency of $n$-3 LC PUFA biosynthesis in mammals, in terms of the metabolic conversion of ALA to EPA or DHA, appears to be quite low, highlighting the desirability of supplementing this endogenous biosynthetic route with dietary LC PUFA. This metabolic conversion appears to be particularly critical at various stages of human life; for example, LC PUFA are important in the development of ocular vision in infants and may also play a role in aspects of neurological and brain development (Gil et al. 2003), while at the other end of the human lifespan these same LC PUFA may help alleviate a number of symptoms associated with geriatric patients (Klaver \& Allikmets, 2003; Leonard et al. 2004).

As mentioned earlier, the primary dietary source of $n-3$ LC PUFA is fish and their associated oils. Marine fish have a similar inefficient $n-3$ LC PUFA biosynthetic pathway to that found in mammals, and also depend on the dietary acquisition of these fatty acids to supplement their endogenous synthesis (Tocher \& Ghioni, 1999). However, the aquatic environment is rich in micro-organisms that synthesise (with high efficiency) n-3 LC PUFA such as EPA and DHA (Napier, 2002). Thus, it is through the consumption of these $n-3$ LC PUFA-rich microbes that fish accumulate (either directly or via carnivorous consumption of other fish) EPA and DHA, rather than through any innate capacity for their synthesis. This progression of $n-3$ LC PUFA up the aquatic food web has implications for attempts to supplement fish stocks by aquaculture (i.e. fish farming), not least of all because farmed fish still require dietary n-3 LC PUFA (Opsahl-Ferstad et al. 2003). Aquaculture is currently the largest consumer of fishderived oils, and so is clearly not capable of operating in a sustainable manner. Conversely, the greater part of man's dietary fatty acid intake is in the form of plant-derived 'vegetable' oils rich in LA and ALA. Since higher plants lack the capacity to synthesise (either $n-6$ or $n-3$ ) LC PUFA, oils derived from them are devoid of fatty acids such as EPA and DHA. It is for this reason that vegetable oils cannot substitute for fish oils in aquaculture (Sargent \& Tacon, 1999). Moreover, most plant oils are rich in $n-6$ fatty acids such as LA, rather than the $n$-3 ALA. The preponderance of vegetable oils in the modern diet has resulted in a dietary 'flood' of $n-6$ fatty acids, with a $n-6: n-3$ of $>10: 1$. This situation contrasts considerably with the human diet of approximately 150 years ago, which probably reflected a ratio of $2: 1$, and was more likely to contain fish oils rich in $n-3$ LC PUFA (Simopoulos, 1999).

Considering all these factors, it seems that there is ample evidence for the health-beneficial properties of dietary consumption of $n-3$ LC PUFA such as EPA and DHA. Unfortunately, just as the importance of $n-3$ LC PUFA is finally being recognised by health protection agencies and the general public at large, the natural resources that provide these oils are in danger of being exhausted. For example, stocks of common marine fish in northern Europe have been over-exploited for decades, resulting in ever-reducing catch size (in terms of both numbers and maturity of fish; Opsahl-Ferstad et al. 2003; Graham et al. 2004). Thus, there is considerable pressure to identify a sustainable source of important fish-oil fatty acids such as DHA and EPA for use in the protection and improvement of human health, in addition to the requirements of aquaculture. This imminent reduction or absence of fish oils should therefore be considered a major driver for seeking and developing alternative sustainable sources of $n-3$ LC PUFA. Approaches using microbiological sources to synthesise LC PUFA for human nutrition have been developed, as exemplified by companies such as Martek Biosciences (Columbia, MD, USA), which specialises in the production of these fatty acids from natural sources such as Crypthecodinium cohnii (an alga rich in DHA) and Mortierella alpina (a fungus rich in arachidonic acid $(20: 4 n-6 ;$ ARA)), by fermentation (i.e. contained culture) of these micro-organisms. However, although this approach allows a rigorous control of the growth of the LC PUFA sources, it also requires the appropriate microbiological facilities.

An alternative production system for the synthesis of LC PUFA is to use plants as 'factories' to accumulate these fatty acids in the storage lipids of their seeds (Thelen \& Ohlrogge, 2002; Jaworski \& Cahoon, 2003; Tucker, 2003). However, since higher plants do not accumulate these fatty acids, LC PUFA biosynthetic genes need to be introduced into a suitable oilseed crop by genetic engineering. The 
Conventional $\Delta^{6}$-desaturase-elongase pathway

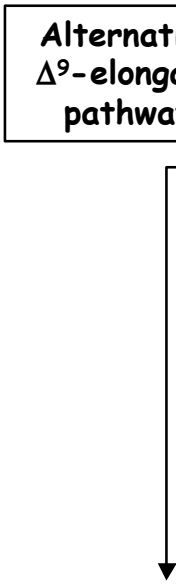

$n-6$

18: 2 Linoleic acid

¿ $\Delta^{6}$-Desaturase

$18: 3 \gamma$-Linolenic acid

П $\Delta^{6}$-Elongase

20: 3 Di-homo $\gamma$-linolenic acid

凸 $\Delta^{5}$-Desaturase

20:4 Arachidonic acid

$18: 2$ Linoleic acid

П $\Delta^{9}$-Elongase

$20: 2$ Eicosadienoic acid

凹 $\Delta^{8}$-Desaturase

20: 3 Di-homo $\gamma$-linolenic acid

』 $\Delta^{5}$-Desaturase

20:4 Arachidonic acid $n-3$

$18: 3 \alpha$-Linolenic acid

凸 $\Delta^{6}$-Desaturase

18:4 Octadecatetraenoic acid

ص $\Delta^{6}$-Elongase

20:4 Eicosatetraenoic acid

』 $\Delta^{5}$-Desaturase

20: 5 Eicosapentaenoic acid

П $\Delta^{5}$-Elongase

22: 5 Docosapentaenoic acid

凸 $\Delta^{7}$-Elongase

24:5 Tetracosapentaenoic acid

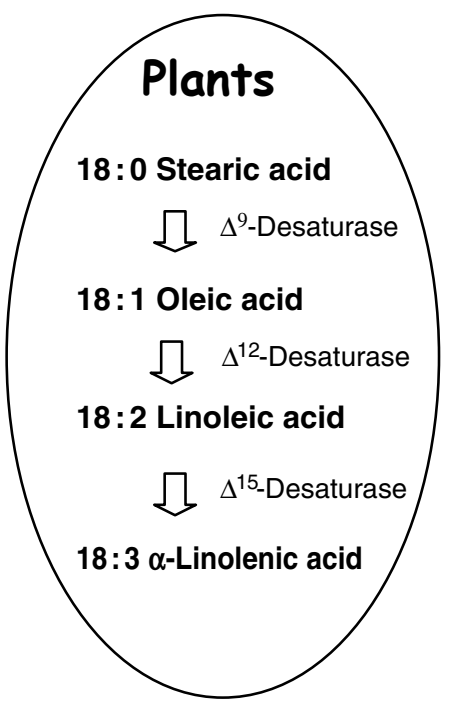

$\Delta^{4}$ - Desaturase pathway

22: 5 Docosapentaenoic acid

』 $\Delta^{4}$-Desaturase

22: 6 Docosahexaenoic acid

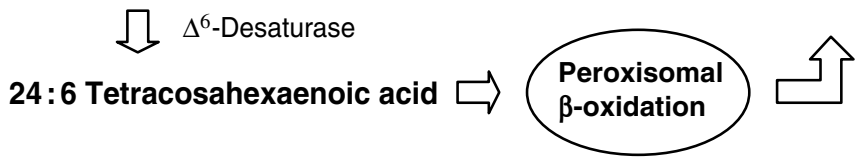

'Sprecher' pathway

Fig. 1. Generalised representation of the biosynthesis of long-chain PUFA. The conventional $\Delta^{6}$-desaturase-elongase pathway for the synthesis of arachidonic acid and EPA from the essential fatty acids linoleic acid and $\alpha$-linolenic acid (synthesised in plants) is shown, as is the alternative $\Delta^{9}$-elongase route (for simplicity, only the $n-6$ side of the alternative pathway is shown, although the $n-3$ substrate $\alpha$-linolenic acid is also metabolised by this route). The $\Delta^{5}$-elongase- $\Delta^{4}$-desaturase route (prevalent in microalgae) for DHA is also indicated, as is the mammalian 'Sprecher' route that involves $\beta$-oxidation.

resultant transgenic plants would thus contain the 'trait' for LC PUFA synthesis, providing a cheap and sustainable source of these important fatty acids (Abbadi et al. 2001; Sayanova \& Napier, 2004). Moreover, since plants are effectively $\mathrm{C}$ neutral, this production process is environmentally sound, utilising only solar energy and $\mathrm{CO}_{2}$. The focus of the present paper will therefore be on recent attempts to produce transgenic plants that synthesise LC PUFA.

\section{The metabolic engineering of transgenic plants} for long-chain PUFA biosynthesis

The production of LC PUFA in transgenic plants requires the heterologous reconstitution of the biosynthetic pathway in the new host. This process in turn depends on the identification and functional characterisation of the genes encoding this biosynthetic pathway in the LC PUFAsynthesising organism, allowing the transfer of these genes to the new host. The last few years has seen considerable progress in the identification and functional characterisation of the genes encoding LC PUFA biosynthetic enzymes (a schematic representation of the biosynthetic pathways is shown in Fig. 1), including all the microsomal fatty acid desaturases that underpin LC PUFA biosynthesis (such as the $\Delta^{6}-, \Delta^{5}-, \Delta^{4}$ - and alternative pathway $\Delta^{8}$-desaturases). All these LC PUFA desaturases belong to the N-terminal cytochrome $b_{5}$ fusion superfamily, and the presence of this N-terminal electron transport domain may be associated with the 'front-end' desaturation process catalysed by these enzymes (unlike the non-fusion microsomal methyldirected desaturases found in higher plants and some lower organisms). Examples of LC PUFA desaturases have been identified from animals, fungi, algae and some of the few plant species (e.g. Boraginaceae, Primulaceae, Oenothera spp.) that carry out limited PUFA desaturation. Many of these desaturases have been functionally characterised in heterologous hosts such as yeast and transgenic plants, and have been observed to function efficiently in non-native hosts (for recent reviews of progress in the characterisation 
of LC PUFA desaturases, see Napier et al. 2003; Sperling et al. 2003).

The second key enzymic reaction in the synthesis of LC PUFA is microsomal acyl-elongation, in which a $\mathrm{C}_{18}$ PUFA substrate (usually containing a 'front-end' $\Delta^{6}$-desaturation) is $\mathrm{C}_{2}$-elongated to yield the $\mathrm{C}_{20} \mathrm{LC}$ PUFA (Fig. 1). Progress on the identification of this microsomal 'elongase' has been hindered by the fact that this elongation process actually comprises four distinct and sequential enzymes, i.e. condensation (of malonyl-CoA and the PUFA acyl-CoA), $\beta$-ketoreduction, dehydration and enoyl reduction. Thus, the question arose as to whether all four components of the microsomal elongase would be required to reconstitute the elongating activity. However, open reading frames from Caenorhabditis elegans (a PUFA-synthesising nematode) and $M$. alpina have been identified by 'gain-of-function' (i.e. acquisition of the ability to elongate $\mathrm{C}_{18}$ PUFA) experiments in yeast (Beaudoin et al. 2000; Parker-Barnes et al. 2000). The identified nematode and fungal open reading frames show homology to the yeast elongation (termed ELO) genes, which are required for the synthesis of (saturated) LC fatty acids found in sphingolipids. Perhaps one of the more surprising observations in the identification of the ELOlike PUFA-elongating activities has been that the expression of these individual open reading frames is still able to reconstitute a PUFA-specific elongase; there is no requirement for the co-expression of any other components of the elongase. This finding is likely to indicate that the initial condensing enzyme (in the form of the ELO-like open reading frames) confers specificity on the elongase, with the other three reactions acting in a non-specific manner. Thus, these data indicate the possibility of re-directing or 'hijacking' an endogenous non-PUFA elongase by the expression of a heterologous PUFA-condensing enzyme and hence, the possibility of transgenic plants engineered to synthesise LC PUFA.

It is also important to consider the various biochemical nuances that exist in LC PUFA biosynthesis, as a number of 'variations on a theme' exist in nature; this topic has been the subject of several recent reviews (Drexler et al. 2003; Sayanova \& Napier, 2004) and will therefore only be summarised here. The predominant LC PUFA biosynthetic pathway has the $\mathrm{C}_{18} \Delta^{6}$-desaturase as the first key committed step, producing $\gamma$-linoleic acid (18:3n-6) and stearidonic acid $(18: 4 n-3)$ from LA and ALA respectively. These $\mathrm{C}_{18} \Delta^{6}$-desaturated products then undergo a $\mathrm{C}_{2}$ elongation (catalysed by the $\Delta^{6}$-elongase) to yield $\mathrm{C}_{20}$ substrates for subsequent $\Delta^{5}$-desaturation to generate ARA and EPA (Fig. 1). Further elongation and desaturation can then produce DHA by two distinct pathways; either via $\Delta^{4}$-desaturation of a $\mathrm{C}_{22}$ elongation product, or the more complicated 'Sprecher' route involving peroxisomal $\beta$-oxidation (Qui, 2003). It appears that whilst the latter route is prevalent in animals, the simpler $\Delta^{4}$-desaturation pathway is present in aquatic microorganisms. One additional important variation in the synthesis of ARA and EPA is observed in some organisms, in which an 'alternative' pathway appears to operate. Here, the order of the initial desaturation and elongation steps are reversed, with a $\mathrm{C}_{18} \Delta^{9}$-specific elongase first synthesising
$\mathrm{C}_{20}$ PUFA, which then undergo $\Delta^{8}$-desaturation (Qi et al. 2002). The resulting products are then subject to conventional $\Delta^{5}$-desaturation to yield ARA and EPA (Fig. 1). As mentioned earlier, all the genes encoding enzymes of the conventional and alternative ARA-EPA pathway have been cloned, as have the genes for the $\Delta^{4}$-desaturation pathway for DHA synthesis.

Initial attempts to reconstitute the LC PUFA biosynthetic pathway in a heterologous host have utilised yeast as a simple model system. Beaudoin et al. (2000) have co-expressed the $C$. elegans $\mathrm{C}_{18^{-}} \Delta^{6}$-elongating activity, polyunsaturated elongating activity-1, with the borage (Borago officinalis) $\Delta^{6}$-desaturase and the $M$. alpina $\Delta^{5}$-desaturase. This approach has resulted in low but marked levels of ARA or EPA in the transgenic yeast, depending on the exogenous substrate $(n-6 v . n-3)$. Similar data have been obtained by co-expressing desaturases from the diatom Phaeodactylum triconutum with a moss $\Delta^{6}$-elongase (Domergue et al. 2002). These authors have noted that as well as a relatively inefficient reconstitution of LC PUFA synthesis, there are a number of so-called 'side-reactions' (i.e. unexpected desaturation or elongation products). More recently, a very detailed analysis of the biochemical steps involved in heterologous LC PUFA biosynthesis in yeast has been carried out, revealing a number of potential bottlenecks in the conventional $\Delta^{6}$-desaturase-elongase pathway (Domergue et al. 2003). This analysis has indicated that one of the major constraints in this pathway may be the different substrate requirements for desaturases or elongases; most nonanimal microsomal PUFA desaturases use glycerolipidlinked substrates (i.e. fatty acids esterified to membrane lipids such as phosphatidylcholine), but fatty acid elongation requires acyl-CoA substrates. Thus, there is a requirement for acyl-exchange between glycerolipids and the acyl-CoA pool; this reaction itself is mediated by an enzyme(s) that may display selectivity towards its substrates (Beaudoin \& Napier, 2004).

\section{First successful production of $\mathbf{C}_{20}$ long-chain PUFA in transgenic plants}

Two studies on the heterologous synthesis of LC PUFA in plants have recently demonstrated the possibility of using transgenic plants to synthesise these important fatty acids. The first study utilised the alternative $\Delta^{9}$-elongase pathway, after the serendipitous isolation of a $\mathrm{C}_{18}$ $\Delta^{9}$-elongating activity from the marine microalga Isochrysis galbana (Qi et al. 2002). On the other hand Wallis \& Browse (1999) have identified the specific $\Delta^{8}$-desaturase activity associated with this pathway from Euglena. The presence of the alternative pathway in Isochrysis was unexpected and, because such a $\Delta^{9}$-elongating activity would utilise endogenous LA and ALA as substrates (in the form of $\mathrm{CoA}$ ), expression of this Isochrysis gene in transgenic plants has provided an opportunity to assess the function of a heterologous PUFA elongase alone.

Expression of the Isochrysis $\Delta^{9}$-elongase in transgenic arabidopsis, using the constitutive CMV 35 S promoter, has resulted in comparatively high levels (approximately $15 \%$ 
total fatty acids) of the $\mathrm{C}_{20}$ PUFA eicosadienoic acid $(20: 2 n-6)$ and eicosatrienoic acid (20:3n-3), through the elongation of LA and ALA, depending on the tissue type (Qi et al. 2004). However, values for $n-6: n-3$ elongation products do not exactly mirror the total levels of endogenous substrates LA and ALA, which probably reflects the extent of acyl exchange between the microsomal 'eukaryotic' and the plastidic 'prokaryotic' pathways in different tissue types (for a full discussion of these data, see Napier et al. 2004). Interestingly, the accumulation of $\mathrm{C}_{20}$ di- and trienoic fatty acids does not cause any perturbation to the morphology or viability of the transgenic arabidopsis plants, which is in contrast with the ectopic expression of the seed-specific fatty acid elongase 1 elongating activity. This latter enzyme directs the elongation of oleic acid to 20:1 (n-9) and 22:1 (n-9) and, in transgenic arabidopsis plants accumulating $>10 \%$ $\mathrm{C}_{20-22}$ monounsaturates, results in dramatic morphological alterations including perturbed plastid membranes (Millar et al. 1998).

To fully reconstitute the alternative $\mathrm{C}_{20}$ PUFA biosynthetic pathway, transgenic arabidopsis lines expressing the Isochrysis $\Delta^{9}$-elongase have been additionally sequentially transformed with the Euglena $\mathrm{C}_{20} \Delta^{8}$-desaturase and the M. alpina $\Delta^{5}$-desaturase. This process results in the conversion of the elongation products 20:2 (n-6) and $20: 3(n-3)$ to $20: 3(n-6)$ and $20: 4(n-3)$ via $\Delta^{8}$-desaturation, and subsequent $\Delta^{5}$-desaturation of these products to ARA and EPA respectively (Qi et al. 2004). These two LC PUFA accumulate in leaf tissues of transgenic arabidopsis plants to a combined level of approximately $10 \%$ total fatty acids, the majority being ARA $(n-6)$. Again, this outcome does not reflect the levels of $n-6: n-3$ substrates (predominantly ALA $(n-3)$ in vegetative tissue). In addition to accumulation of ARA and EPA, several other $\mathrm{C}_{20}$ PUFA were also detected and identified as sciadonic acid $\left(20: 3 \Delta^{5,11,14}\right)$ and juniperonic acid $\left(20: 4 \Delta^{5,11,14,17}\right)$. These two PUFA are non-methylene-interrupted and appear to have been synthesised as a result of the 'promiscuous' activity of the $\Delta^{5}$-desaturase. Whether this outcome represents a perturbation of substrate channelling in the reconstituted alternative LC PUFA biosynthetic pathway remains to be determined, but it is worth noting that the enzyme used in this study (the $M$. alpina $\Delta^{5}$-desaturase) has previously been observed to synthesise unusual $\Delta^{5}$-desaturated $\mathrm{C}_{18}$ fatty acids when individually expressed in transgenic rapeseed (Knutzon et al. 1998). Whilst sciadonic and juniperonic acids were not primary targets for the synthesis and accumulation in transgenic plants, recent evidence suggests that these LC PUFA may also be beneficial to health and may play a role in modulating some aspects of human metabolism. In particular, 2-monoacylglycerol containing sciadonic acid has been observed to act as a ligand for the human $\mathrm{CB}_{1}$ cannabinoid receptor (Nakane et al. 2000). Both sciadonic and juniperonic acids are found in a number of species of pine seeds, and as such have been consumed by human subjects without demonstrating any anti-nutritional effects (Berger et al. 2002).

The use of the alternative $\Delta^{9}$-elongase- $\Delta^{8}$-desaturase to successfully reconstitute LC PUFA biosynthesis in transgenic plants has been recognised as an important breakthrough in the production of these compounds in a sustainable manner (Green, 2004; Napier et al. 2004). However, whilst these current data represent a 'proof-ofconcept' demonstration, the results of seed-specific expression of this pathway in a transgenic oilseed crop are still eagerly awaited.

Thus, the studies of Abbadi et al. (2004) on the expression of the $\Delta^{6}$-desaturase-elongase pathway provide some further insights into the feasibility and constraints on LC PUFA synthesis in transgenic oilseeds. Using enzyme activities from a number of different PUFA-accumulating species, transgenic linseed and tobacco lines have been generated that express the $\Delta^{6}$-desaturase, the $\Delta^{6}$-elongase and the $\Delta^{5}$-desaturase. These three activities have been placed under the transcriptional regulation of seed-specific promoters and introduced into the transgenic plant as a single integration event. Analysis of the seeds of transgenic tobacco or linseed has revealed very high levels of $\Delta^{6}$-desaturated fatty acids yet only relatively low amounts of ARA and EPA. Whilst these data clearly demonstrate the reconstitution of the 'conventional' PUFA biosynthetic pathway in transgenic seeds, they also parallel earlier observations in yeast on the efficient synthesis of $\mathrm{C}_{20}$ PUFA (Beaudoin et al. 2000; Domergue et al. 2002, 2003). Further detailed analysis of transgenic linseed expressing these activities has revealed a number of subtle observations (Abbadi et al. 2004). First, although the $\Delta^{6}$ desaturase and the $\Delta^{6}$-elongase appear to function at very different rates, the two transgenes are transcribed at similar levels. Second, although the $\Delta^{6}$-desaturated substrates for elongation accumulate at high levels in the microsomal membranes, particularly at the $s n-2$ position of phosphatidylcholine, this outcome is not reflected by a concomitant increase in the $\Delta^{6}$-desaturated acyl-CoA; thus, there is inefficient exchange from phosphatidylcholine into the acyl-CoA pool. As an additional (and converse) complication, $\mathrm{C}_{18} n-3$ fatty acids appear to be very efficiently channelled from phosphatidylcholine into triacylglycerols (most probably via the action of an acyl-CoA-independent acyltransferase activity), by-passing the site of elongation and effectively precluding them from further modification (Abbadi et al. 2004; Beaudoin \& Napier, 2004; Napier et al. 2004).

Taking these observations together, it seems likely that a major constraint on the synthesis of LC PUFA via the conventional $\Delta^{6}$-desaturase-elongase route is the dichotomy of substrate requirements exhibited by the glycerolipids desaturases and the acyl-CoA microsomal elongase. However, the levels obtained of ARA and EPA in the seeds of transgenic linseed are still reasonable, even allowing for the clearly suboptimal exchange and channelling of acyl substrates. Thus, these results should be taken as highly encouraging for the successful synthesis of LC PUFA via this pathway.

\section{Future prospects: towards the production of DHA}

The accumulation of $\mathrm{C}_{20}$ LC PUFA ARA and EPA in transgenic plants expressing either the conventional or alternative desaturase-elongase pathways is a major 
achievement, and whilst the levels are relatively modest (approximately 3\% EPA), they still represent levels that could provide nutritional enhancement to animal diets. However, an additional target for the alternative sustainable production of 'fish oils' in transgenic oilseeds is the synthesis and accumulation of DHA. The simplest route for DHA synthesis is by the $\mathrm{C}_{2}$ elongation of EPA, via the action of a $\Delta^{5}$-elongase (to elongate EPA to docosapentaenoic acid (22:5n-3)) and subsequent desaturation with the $\mathrm{C}_{22} \Delta^{4}$-desaturase (Qiu, 2003). Thus, two additional transgenes need to be introduced into transgenic plants that already accumulate EPA. The cytochrome $b_{5}$-fusion $\mathrm{C}_{22} \Delta^{4}$-desaturase has been identified and functionally characterised from several aquatic micro-organisms (Qiu et al. 2001). More recently, the specific elongating activity required for the synthesis of 22:5 (n-3) from EPA has been characterised (Meyer et al. 2004; Pereira et al. 2004). However, it might be expected that the previouslymentioned constraints on efficient elongation associated with poor acyl exchange between glycerolipids and the acyl-CoA pool might also apply to the synthesis of DHA, with a potential bottleneck occurring via the accumulation of EPA in phosphatidylcholine (see Fig. 1). As also noted earlier, the expression of the LC PUFA biosynthetic pathway in transgenic plants results in the synthesis of both $n-6$ and $n-3$ products, rather than just the predominantly $n-3$ LC PUFA found in fish oils. Thus, more efficient channelling (or re-routing) of both substrates and products will be required to optimise the heterologous reconstitution of $n-3$ LC PUFA synthesis in plants. Thus, it will be of considerable interest to assess the possibility of using the non-aerobic polyketide synthase-like system to synthesise DHA in transgenic plants, since this biosynthetic route does not apparently require acyl-exchange (at least during the processive synthesis of DHA; Metz et al. 2001; Napier, 2002).

\section{Conclusions}

The possibility of 'reverse engineering' LC PUFA biosynthesis into transgenic plants has now been successfully demonstrated for ARA and EPA. These important breakthroughs have also provided new insights into the maintenance of lipid homeostasis in plants, and the key role of acyl-exchange enzymes in LC PUFA reconstitution. This additional knowledge will underpin future attempts to engineer the synthesis of $\mathrm{C}_{22}$ LC PUFA such as DHA. It is therefore to be hoped that the use of transgenic plants as a source of fish oils for human health and nutrition can be considered as a sustainable alternative to the diminishing marine fish stocks (Graham et al. 2004). It should also be remembered that since aquaculture is also dependent on fish oils for the correct nutrition of farmed fish species, transgenic-derived plant oils enhanced by the presence of LC PUFA may also be able to sustainably replace this additional demand (Opsahl-Ferstad et al. 2003).

\section{Acknowledgements}

Rothamsted Research receives grant-aided support from the Biotechnology and Biological Sciences Research
Council (BBSRC) UK. The authors thank BASF Plant Sciences for support. Current research is supported by the EU Sixth Framework Programme Integrated Project LIPGENE (contract no. FOOD-CT-2003-505944).

\section{References}

Abbadi A, Domergue F, Fahl A, Ott C, Bauer J, Napier JA, Welti R, Cirpus P \& Heinz E (2004) Biosynthesis of very long chain polyunsaturated fatty acids in transgenic oilseeds: constraints on their accumulation. Plant Cell 16, 2734-2748.

Abbadi A, Domergue F, Meyer A, Riedel K, Sperling P, Zank T \& Heinz E (2001) Transgenic oilseeds as sustainable source of nutritionally relevant $\mathrm{C}_{20}$ and $\mathrm{C}_{22}$ polyunsaturated fatty acids? European Journal of Lipid Science and Technology 103, 106-113.

Bang HO, Dyerberg J \& Hjoorne N (1976) The composition of food consumed by Greenland Eskimos. Acta Medica Scandinavica 200, 69-73.

Beaudoin F, Michaelson LV, Hey SJ, Lewis MJ, Shewry PR, Sayanova O \& Napier JA (2000) Heterologous reconstitution in yeast of the polyunsaturated fatty acid biosynthetic pathway. Proceedings of the National Academy of Sciences USA 97, 6421-6426.

Beaudoin F \& Napier JA (2004) Biosynthesis and compartmentalisation of triacyglycerol in higher plants. Topics in Current Genetics 6, 267-287.

Berger A, Monnard I, Baur M, Charbonnet C, Safonova I \& Jomard A (2002) Epidermal anti-inflammatory properties of 5,11,14, 20:3: Effects on mouse ear edema, PGE2 levels in cultured keratinocytes, and PPAR activation. Lipids in Health and Disease 1, 1-12.

Burr ML, Fehily AM, Gilbert JF, Rogers S, Holliday RM, Sweetnam PM, Elwood PC \& Deadman NM (1989) Effects of changes in fat, fish, and fibre intakes on death and myocardial reinfarction: diet and reinfarction trial (DART). Lancet ii, 757-761.

Domergue F, Abbadi A, Ott C, Zank TK, Zahringer U \& Heinz E (2003) Acyl carriers used as substrates by the desaturases and elongases involved in very long-chain polyunsaturated fatty acids biosynthesis reconstituted in yeast. Journal of Biological Chemistry 278, 35115-35126.

Domergue F, Lerchl J, Zahringer U \& Heinz E (2002) Cloning and functional characterization of Phaeodactylum tricornutum front-end desaturases involved in eicosapentaenoic acid biosynthesis. European Journal of Biochemistry 269, 4105-4113.

Drexler H, Spiekermann P, Meyer A, Domergue F, Zank T, Sperling P, Abbadi A \& Heinz E (2003) Metabolic engineering of fatty acids for breeding of new oilseed crops: strategies, problems and first results. Journal of Plant Physiology 160, 779-802.

Dyerberg J \& Bang HO (1982) A hypothesis on the development of acute myocardial infarction in Greenlanders. Scandinavian Journal of Clinical and Laboratory Investigation Suppl., 161, $7-13$.

German JB, Roberts MA \& Watkins SM (2003) Genomics and metabolomics as markers for the interaction of diet and health: lessons from lipids. Journal of Nutrition 133, 2078S-2083S.

Gil A, Ramirez M \& Gil M (2003) Role of long-chain polyunsaturated fatty acids in infant nutrition. European Journal of Clinical Nutrition 57, Suppl. 1, S31-S34.

GISSI-Prevenzione Investigators (1999) Dietary supplementation with $n-3$ polyunsaturated fatty acids and vitamin $\mathrm{E}$ after myocardial infarction: results of the GISSI-Prevenzione trial. Gruppo Italiano per lo Studio della Sopravvivenza nell'Infarto miocardico. Lancet 354, 447-455. 
Go VL, Butrum RR \& Wong DA (2003) Diet, nutrition, and cancer prevention: the postgenomic era. Journal of Nutrition 133, 3830S-3836S.

Graham IA, Cirpus P, Rein D \& Napier JA (2004) The use of very long chain polyunsaturated fatty acids to ameliorate Metabolic Syndrome: transgenic plants as an alternative sustainable source to fish oils. Nutrition Bulletin 29, 228-233.

Green AG (2004) From alpha to omega-producing essential fatty acids in plants. Nature Biotechnology 22, 680-682.

Hu FB, Manson JE \& Willett WC (2001) Types of dietary fat and risk of coronary heart disease: a critical review. Journal of the American College of Nutrition 20, 5-19.

Jaworski J \& Cahoon EB (2003) Industrial oils from transgenic plants. Current Opinion in Plant Biology 6, 178-184.

Klaver CC \& Allikmets R (2003) Genetics of macular dystrophies and implications for age-related macular degeneration. Developments in Ophthalmology 37, 155-169.

Knutzon DS, Thurmond JM, Huang YS, Chaudhary S, Bobik EG Jr, Chan GM, Kirchner SM \& Mukerji P (1998) Identification of $\Delta 5$-desaturase from Mortierella alpina by heterologous expression in Bakers' yeast and canola. Journal of Biological Chemistry 273, 29360-29366.

Leonard AE, Pereira SL, Sprecher H \& Huang YS (2004) Elongation of long-chain fatty acids. Progress in Lipid Research 43, 36-54.

Metz JG, Roessler P, Facciotti D, Levering C, Dittrich F, Lassner M et al. (2001) Production of polyunsaturated fatty acids by polyketide synthases in both prokaryotes and eukaryotes. Science 293, 290-293.

Meyer A, Kirsch H, Domergue F, Abbadi A, Sperling P, Bauer J et al. (2004) Novel fatty acid elongases and their use for the reconstitution of docosahexaenoic acid biosynthesis. Journal of Lipid Research 45, 1899-909.

Millar AA, Wrischer M \& Kunst L (1998) Accumulation of verylong-chain fatty acids in membrane glycerolipids is associated with dramatic alterations in plant morphology. Plant Cell $\mathbf{1 0}$, 1889-1902.

Nakane S, Tanaka T, Satouchi K, Kobayashi Y, Waku K \& Sugiura $T$ (2000) Occurrence of a novel cannabimimetic molecule 2-sciadonoylglycerol (2-eicosa-5',11',14'-trienoylglycerol) in the umbrella pine Sciadopitys verticillata seeds. Biological and Pharmaceutical Bulletin 23, 758-761.

Napier JA (2002) Plumbing the depths of PUFA biosynthesis: a novel polyketide synthase-like pathway from marine organisms. Trends in Plant Science 7, 51-54.

Napier JA, Michaelson LV \& Sayanova O (2003) The role of cytochrome $\mathrm{b}_{5}$ fusion desaturases in the synthesis of polyunsaturated fatty acids. Prostaglandins, Leukotrienes, and Essential Fatty Acids 68, 135-143.

Napier JA, Sayanova O, Qi B \& Lazarus CM (2004) Progress towards the production of polyunsaturated fatty acids in transgenic plants. Lipids 39, 1067-1075.

Nugent AP (2004) The metabolic syndrome. Nutrition Bulletin 29, 36-43.

Opsahl-Ferstad H-G, Rudi H, Ruyter B \& Refstie S (2003) Biotechnological approaches to modify rapeseed oil composition for applications in aquaculture. Plant Science 165, 349-357.

Paoloni-Giacobino A, Grimble R \& Pichard, C (2003) Genetics and nutrition. Clinical Nutrition 22, 429-435.

Parker-Barnes JM, Das T, Bobik E, Leonard AE, Thurmond JM, Chaung LT, Huang YS \& Mukerji P (2000) Identification and characterization of an enzyme involved in the elongation of $n-6$ and $n-3$ polyunsaturated fatty acids. Proceedings of the National Academy of Sciences USA 97, 8284-8289.

Pereira SL, Leonard AE, Huang YS, Chuang LT \& Mukerji P (2004) Identification of two novel microalgal enzymes involved in the conversion of the omega 3-fatty acid, eicosapentaenoic acid (EPA), to docosahexaenoic acid (DHA). Biochemical Journal 384, 357-366.

Qi B, Beaudoin F, Fraser T, Stobart AK, Napier JA \& Lazarus CM (2002) Identification of a cDNA encoding a novel $\mathrm{C}_{18}-\Delta 9$ polyunsaturated fatty acid-specific elongating activity from the docosahexaenoic acid (DHA)-producing microalga, Isochrysis galbana. FEBS Letters 510, 159-165.

Qi B, Fraser T, Mugford S, Dobson G, Sayanova O, Butler J, Napier JA, Stobart AK \& Lazarus CM (2004) The production of very long chain polyunsaturated omega- 3 and omega- 6 fatty acids in transgenic plants. Nature Biotechnology 22, 739-745.

Qiu X (2003) Biosynthesis of docosahexaenoic acid (DHA, 22:6-4. 7,10,13,16,19): two distinct pathways. Prostaglandins, Leukotrienes, and Essential Fatty Acids 68, 181-186.

Qiu X, Hong H \& MacKenzie SL (2001) Identification of a $\Delta 4$ fatty acid desaturase from Thraustochytrium sp. involved in the biosynthesis of docosahexanoic acid by heterologous expression in Saccharomyces cerevisiae and Brassica juncea. Journal of Biological Chemistry 276, 31561-31566.

Sargent JR \& Tacon AG (1999) Development of farmed fish: a nutritionally necessary alternative to meat. Proceedings of the Nutrition Society 58, 377-383.

Sayanova O \& Napier JA (2004) Eicosapentaenoic acid: biosynthetic routes and the potential for synthesis in transgenic plants. Phytochemistry 65, 147-158.

Shastry BS (2002) SNP alleles in human disease and evolution. Journal of Human Genetics 47, 561-566.

Simopoulos AP (1999) Essential fatty acids in health and chronic disease. American Journal of Clinical Nutrition $\mathbf{7 0}$, 560S-569S.

Simopoulos AP (2000) Human requirement for $n-3$ polyunsaturated fatty acids. Poultry Science 79, 961-970.

Sperling P, Ternes P, Zank TK \& Heinz E (2003) The evolution of desaturases. Prostaglandins, Leukotrienes, and Essential Fatty Acids 68, 73-95.

Thelen JJ \& Ohlrogge JB (2002) Metabolic engineering of fatty acid biosynthesis in plants. Metabolic Engineering 4, 12-21.

Tocher DR \& Ghioni C (1999) Fatty acid metabolism in marine fish: low activity of fatty acyl $\Delta 5$ desaturation in gilthead sea bream (Sparus aurata) cells. Lipids 34, 433-440.

Tucker G (2003) Nutritional enhancement of plants. Current Opinion in Biotechnology 14, 221-225.

von Schacky C (2003) The role of omega-3 fatty acids in cardiovascular disease. Current Atherosclerosis Reports 5, 139-145.

Wallis JG \& Browse J (1999) The $\Delta 8$-desaturase of Euglena gracilis: an alternate pathway for synthesis of 20-carbon polyunsaturated fatty acids. Archives of Biochemistry and Biophysics 365, 307-316.

Wallis JG, Watts JL \& Browse J (2002) Polyunsaturated fatty acid synthesis: what will they think of next? Trends in Biochemical Sciences 27, 467-470.

Zhao Z, Fu YX, Hewett-Emmett D \& Boerwinkle E (2003) Investigating single nucleotide polymorphism (SNP) density in the human genome and its implications for molecular evolution. Gene 312, 207-213. 
https://doi.org/10.1079/PNS2005447 Published online by Cambridge University Press 